\title{
Heuristic Algorithm Based Dynamic Scheduling Model of Home Appliances in Smart Grid
}

\author{
Inam Ullah Khan ${ }^{1,2}$, Xiandong Ma ${ }^{1}$, C. James Taylor ${ }^{1}$, Nadeem Javaid ${ }^{3}$, Kelum A.A. Gamage ${ }^{4}$ \\ ${ }^{1}$ Engineering Department, Lancaster University, Lancaster LA1 4YW, UK \\ ${ }^{2}$ Electrical Engineering Department, COMSATS University Lahore, Pakistan \\ ${ }^{3}$ COMSATS University, Islamabad 44000, Pakistan \\ ${ }^{4}$ School of Engineering, University of Glasgow, Glasgow G12 8QQ, UK \\ i.u.khan@lancaster.ac.uk; inamkhan@ciitlahore.edu.pk
}

\begin{abstract}
Smart grid provides an opportunity for customers as well as for utility companies to reduce electricity costs and regulate generation capacity. The success of scheduling algorithms mainly depends upon accurate information exchange between main grids and smart meters. On the other hand, customers are required to schedule loads, respond to energy demand signals, participate in energy bidding and actively monitor energy prices generated by the utility company. Strengthening communication infrastructure between the utility company and consumers can serve the purpose of consumer satisfaction. We propose a heuristic demand side management model for scheduling smart home appliances in an automated manner, to maximise the satisfaction of the consumers associated with it. Simulation results confirm that the proposed hybrid approach has the ability to reduce peak-to-average ratio of the total energy demand and reduce the total cost of the energy without compromising user comfort.
\end{abstract}

Keywords: Demand side management, appliance scheduling, critical peak pricing, household energy management.

\section{INTRODUCTION}

Demand side management (DSM) usually refers to those decisions, which are taken by utility companies at user's premises [1]. DSM programs are initiated to use available energy in a more efficient way without developing new infrastructure for generation, transmission and distribution. DSM programs usually encompass demand response programs, fuel substitution programs, efficient conservation of energy programs and above all commercial or residential load management programs [2][4]. Reducing and shifting consumption is one of the main key design features of the residential load management program [5]. This can only be achieved if users are encouraged to build energy efficient buildings and to be well aware of their energy consumption patterns. A part from this practical initiative needs to be taken, including high power appliances shifting from peak hours to offpeak hours for measurable reduction in peak-to-average ratio (PAR) in load demand. Load shifting is expected to be even more important because of high penetration of the plug-in hybrid electrical vehicles (PHEVs). Usually
PHEVs require $0.2-0.3 \mathrm{kWh}$ charging power for one mile driving [6]. This significantly enhances new load on the existing distribution system. Particularly during charging hours, it doubles average household demand, thus worsening the existing high PAR. In absence of properly reinforced system, a high PHEVs penetration can create unbalanced condition, thus compromising power quality standards, voltage regulation issues and even prospective damage to utility and consumer equipment.

Direct load control (DLC) is another useful approach for residential load management [7]-[9]. By applying DLC programs, utility company remotely controls energy consumption and operations of certain household appliances. For instance, thermal comfort equipment including heating, ventilating and air conditioning (HVAC), refrigerators, pumps and light control are wellknown examples of DLC programs. When considering home automation and residential load control specifically, users' comfort is on the top priority and considered as a hurdle in DLC programs execution [10].

Today dynamic pricing replaces DLC programs features. In dynamic pricing mechanism, users are motivated to manage their loads individually on a voluntary basis, e.g., shutting and shifting heavy loads from peak hours to offpeak hours [11]-[13]. Most popular and frequently used schemes of dynamic pricing includes critical-peak pricing (CPP), real-time pricing (RTP), inclined block rate (IBR), time of use pricing (ToUP) and day ahead pricing (DAP) schemes, etc. With the help of these schemes users are encouraged to shift appliances from peak hour to off-peak hours. This helps to achieve a lower PAR and reduces consumer costs [14].

\section{RELATED WORK}

Researchers have recently developed and implemented different state of the art algorithms in smart grid (SG). These algorithms successfully analyzed commercial, residential and industrial buildings in terms of their load consumption profile. Researchers have focused on optimizing energy controller and scheduler in such a way that energy cost is brought to an optimum level for utility companies and customers. Maximum attention is given to balancing the supply-demand ratio and reducing customer cost to a minimum level. Multiple factors are considered while developing these algorithms: appliance rating, 
pricing schemes, utility company priorities and consumer demand in order to get maximum benefit for all stakeholders.

In reference [9], dynamic price RTP is used for optimally scheduling smart home appliances. They mainly focus on reducing unconventional electricity usage, minimizing cost and maximizing benefits from energy storage. Electricity cost is reduced to $22.6 \%$ and peak price is reduced to $11.7 \%$ as compared to the normal pricing scheme. However, authors do not pay attention towards optimizing scheme in their work. While architecting the DSM model, authors purchase the energy during off-peak hours and store it in storage bank for peak hours use. Their main focus is cost minimization and energy storage in battery bank. Linear programming based optimal scheduling model is proposed; however, goals remain unachieved.

Non-deterministic polynomial-time (NP) hardness based optimal scheduling model is discussed in [12]. The authors use greedy iterative algorithms to meet the home scheduling goal. In their work, optimization is achieved by using linear programming and artificial intelligence optimization approaches. Lower peak load and lower peak fluctuation phenomenon is also discussed. Problem formulation is made not only based on user's load demand but also on the generation cost.

In references [11]-[15], authors propose a mixed integer linear programming based algorithm for scheduling home appliances in a fascinate way. The real price tariff is used for scheduling home appliances to reduce cost as well as peak reduction. In [16], multiple types of users in the proposed model are evaluated. These users are categorized as commercial, industrial and residential users. From simulation results, it can be concluded that the proposed algorithm contributes significantly to minimization of PAR and electricity cost.

Genetic Algorithm (GA) based cost minimization method is used in references [12]-[14]. In these papers, renewable energy sources (RESs) and battery storage are integrated into the existing system. RESs are supposed to charge battery bank for later use, when electricity prices are high during high demand of energy. For battery efficiency and life, a controller is developed to monitor the charging and discharging thresholds associated with the battery bank. Furthermore, when electricity prices are low, batteries are supposed to fully charge. Later, when prices are high then certain high priority appliances are handled from battery source to save user cost.

In this paper, we propose meta-heuristic optimization models based on genetic algorithm, grey wolf optimization (GWO) and a hybrid grey wolf and genetic algorithm $\left(\right.$ hybrid $\mathrm{G}^{2}$ ) for scheduling 12 home appliances. Each day is divided into 96 time slots (each 15 minutes) instead of one-hour time slot for appliance operation. This is necessary because in many cases an appliance requires less than an hour to complete its operation such as the electric cattle and dish washer. In this way, users have much freedom and opportunities to reduce cost, PAR and total energy demand. Finally, simulation results of the unscheduled, GA schedule, GWO and hybrid $G^{2}$ are presented to show the effectiveness of the proposed hybrid $G^{2}$ model for appliance scheduling in DSM.

\section{PROPOSED ARCHITECTURE}

In this work, a smart home with multiple smart appliances is considered. Length of operation time (LoTs) and power rating (PR) information of all appliances are already taken from end consumers. The whole system is divided into three sub-layers, including supply side management layer (SSML), communication management layer (CML) and demand side management layer (DSML). SSML contains all information related to energy generation. DSML uses energy management controller (EMC) and appliance scheduler (AS), and schedules

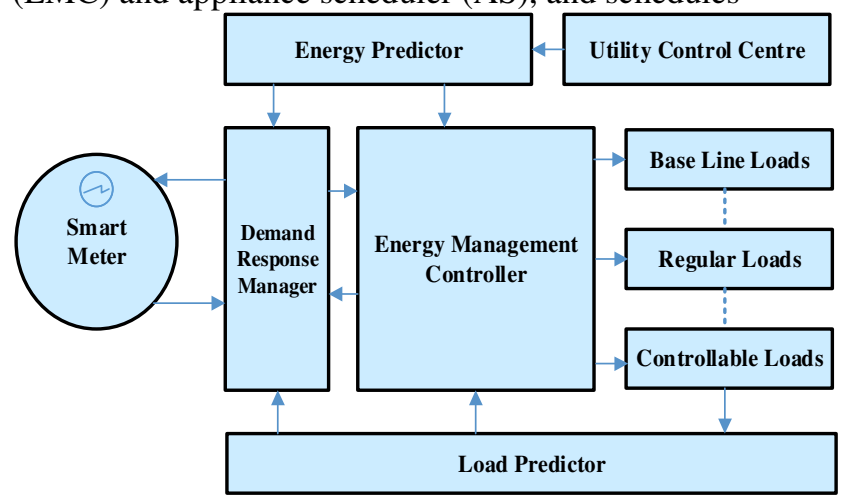

Fig. 1 The proposed system architecture

smart appliances on the basis of LOTs defined by the end users. Purpose of load balancer (LB) is used to delay appliance operation to minimize demand-supply gap and not to allow consumer demand to exceed the limit. Through CML, energy forecaster (EF) and demand response manger (DRM) exchange real-time demandsupply information with SSML and DSML. Home Area Network (HAN) conducts effective communication between EMC through Wi-Fi, Z-wave and Zig-Bee communication protocols. Furthermore, smart appliances are further categorized into base line loads, regular loads and controllable loads depending upon whether their operation can be interrupted or not when activated. EMC uses appliance interface (AI) which controls on/off operation tasks of all smart appliances associated with the system. It is pertinent to mention here that EMC through AS, stops all scheduling operation of the appliances if the interrupt is generated by the consumer to enhance the comfort.

Three meta-heuristic techniques are adopted in this paper, including GA, GWO and hybrid $G^{2}$ in order to schedule these smart appliances in home energy management system (HEMS). Scheduling is performed to save electricity utilization cost for end users. Knapsack problem is formulated to establish coordination among smart appliances at run-time. This gives autonomy to each consumer for managing appliance operation according to the comfort. 


\section{APPLIANCE CATEGORIZATION}

Home appliances are classified into three sub-categories based on their operational behavior. Interruptible appliances are those whose operation can be interrupted or delayed during operation but their operational time is unchangeable. Similarly, uninterruptible appliances are those whose operation cannot be delayed or interrupted once they are in operating mode. However, these appliances can be shifted to other time slots before their operations start.

It is important to shift interruptible and uninterruptible appliances to other time slots to maintain overall energy consumption up to an allowed level. It is beneficial to use the interruptible appliances at low peak hours for saving electricity cost. On contrary, base appliances are those which can neither be interrupted nor deferred in home energy management system (HEMS). For example, refrigerator, air conditioning, lightening and microwave oven are such devices whose operation pattern remains unchanged. All appliances along with their length of operation time (LOT), power rating and category used in this study are listed in Table 1.

In this section, we will analytically describe power system, energy cost and load control model for residential purpose. Based on these descriptions, we will formulate three design optimization problems in next section.

\section{A. Power System}

We consider a smart power system with various load consumers and a single energy source which may be a generator or connection to the main grid through a step down transformer. Furthermore, we assume that each consumer is equipped with EMC that is capable of scheduling different appliances (12 in our model) during different intervals of time (96 intervals in a complete day, i.e., 15 minutes each). By making use of appropriate communication protocol, different smart meters are interconnected, not only with the grid but also with each other by sharing the updated information.

Let $U$ be the set of users, where for each user $u$, let $l_{u}^{t}$ denotes the total load at time slot $t \in T\{1, \ldots \ldots . T\}$, where $T=96$. Daily consumed load by a specific user $u$ is denoted by $l_{u} \in\left[l_{u}^{1}, \ldots \ldots \ldots . . . l_{u}^{T}\right]$. This leads us to calculate the total load of all users in a single time slot across the whole day $t \in T$. It is represented as

$L_{t}=\sum_{u \in U} l_{u}^{t}$

Similarly, daily peak load and average load can be calculated as

$\operatorname{Load}_{\text {peak }}=\operatorname{maximum}_{t \in T} L_{t}$

and

Load $_{\text {average }}=\frac{1}{T} \sum_{t \in T} L_{t}$

From equations (2) and (3), PAR can be calculated as below

$\mathrm{PAR}=\frac{\text { Load }_{\text {peak }}}{\text { Load }_{\text {average }}}=\frac{\text { T maximum }_{\mathrm{t} \in \mathrm{T}} \mathrm{L}_{\mathrm{t}}}{\sum_{\mathrm{t} \in \mathrm{T}} \mathrm{L}_{\mathrm{t}}}$
For each time slot $t \in T$ energy cost for electricity generation or distribution is represented by $C_{t}\left(L_{t}\right)$. Generally, for the same load, cost may differ in different time slot of a day. It mostly depends upon electrical price maintained by the utility at generation site. It is worth mentioning here that cost function being considered in this paper can represent either the original cost of thermal generators or artificial cost tariffs maintained by the utility for proper execution of the load control programs. Actual energy cost function can be represented in terms of a quadratic function in equation (5).

$C_{t}\left(L_{t}\right)=a_{t} L_{t}^{2}+b_{t} L_{t}+c_{t}$

where $a_{t}, b_{t}$ and $c_{t} \geq 0$ at each time slot $\mathrm{t} \in T$

\section{Residential Load Control}

For an individual user $\mathrm{u} \in \mathrm{U}$, let $U_{A}$ denotes the different set of appliances, including base, interruptible and uninterruptible appliances in a home. For scheduling purposes, we initially define a schedule vector for each appliance a $\in A_{n}$ of individual user, where $n$ is the number of the appliances.

$K_{u, a}=\left[K_{u, a}^{1}, \ldots \ldots \ldots \ldots . K_{u, a}^{T}\right]$

where $K_{u, a}^{t}$ represents scheduled energy consumption in one time-slot for appliance $a$ by user $u$. We can then calculate the total load by $u t h$ user.

$l_{u}^{t}=\sum_{a \in A_{n}} k_{u, a}^{t}, \mathrm{t} \in \mathrm{T}$

In our proposed model, main task of AS is to determine an optimum time slot in uth user's smart meter for the individual appliance $a$. In this way, user $u$ can shape its daily load profile by making use of equation (7). It is important to mention here that energy scheduler does not aim to reduce power consumption of different appliances rather it shifts to other different time slots for minimization of PAR and energy cost. Initially, a user needs to initiate start and end time slot in which a particular appliance $a$ is supposed to complete its task. Let beginning time slot be represented by $\alpha_{u, a} \in T$ and end time slot is represented by $\beta_{u, a} \in T$ and $\alpha_{u, a}<\beta_{u, a}$.

Table 1. Appliance parameters

\begin{tabular}{|l|l|l|l|}
\hline Appliances & $\begin{array}{l}\text { Lot } \\
\text { (slots) }\end{array}$ & $\begin{array}{l}\text { Power } \\
\text { rating } \\
(\mathbf{k W h})\end{array}$ & Category \\
& & 1.0 & Uninterruptible \\
\hline Washing machine & 20 & 1.6 & Uninterruptible \\
\hline Clothes dryer & 16 & 2.0 & Interruptible \\
\hline Electric vehicle & 36 & 2.0 & Interruptible \\
\hline Water pump & 32 & 0.5 & Interruptible \\
\hline Humidifier & 12 & 1.5 & Interruptible \\
\hline Vacuum cleaner & 24 & 2.0 & Interruptible \\
\hline Water heater & 48 & 1.2 & Interruptible \\
\hline Dish washer & 16 & 1.4 & Base \\
\hline Refrigerator & 96 & 1.5 & Base \\
\hline Air conditioner & 40 & 0.8 & Base \\
\hline Light & 52 & 2.0 & Base \\
\hline Microwave oven & 16 & &
\end{tabular}

\section{B. Energy Cost Model}


For example, an electrical vehicle (EV) having $E_{u, a}=$ $2 \mathrm{kWh}$ needs 4 hours to complete its charging cycle for 50 $\mathrm{km}$ driving range in a single day. For compiling task, a user must select a larger time slot because in case of any interruption, scheduler completes the task by its end time. For example, the user may select $\alpha_{u, a}=$ 12 a.m. and $\beta_{u, a}=8$ a.m.. Mathematically, it is represented as

$\sum_{t=\alpha_{u, a}}^{\beta_{u, a}} x_{u, a}^{t}=E_{u, a}$

where $x_{u, a}^{t}$ represents energy consumption vector of appliance $a$ during $t$ time slot by $u$. Also, from equation (8), it is concluded that appliance $a$ schedules balances according to daily consumption requirement. Similarly, total energy consumption by all appliances and by all users can be summed up.

$\sum_{t \in T} L_{t}=\sum_{u \in U} \sum_{a \in A_{n}} E_{u, a}$

Since electronic devices are divided into base, interruptible and uninterruptible smart appliances, so in case of uninterruptible appliances strict energy consumption needs to be adopted. In our case, washing machine (WM) and clothes dryer $(\mathrm{CD})$ have constraints that once WM task ends, CD must start its operation immediately. In that case, $\alpha_{u, a}=1$ for $\mathrm{WM}$ and $\beta_{u, a}=0$ for CD. Similarly, a refrigerator is on all the time, so in that case $\alpha_{u, a}=1$ for $\mathrm{WM}$ and $\beta_{u, a}=96$. Generally, a scheduler has no active impact on the operation of the non-interruptible appliances. For a complete energy consumption profile, standby power of interruptible appliances needs to be calculated. It is the power which is consumed by interruptible appliances when they are in idle mode. we need to calculate minimum $\left(\gamma_{u, a}^{\text {Minimum }}\right)$ and maximum $\left(\gamma_{u, a}^{\text {Maximum }}\right)$ standby power level for interruptible appliances. Standby power can be assumed to be such power which a device is consuming when it is in non operation mode but ready to start its operation. We can assume it as:

$\gamma_{u, a}^{\text {Minimum }} \geq x_{u, a}^{t} \geq \gamma_{u, a}^{\text {Maximum }}$

We are now ready to calculate different optimal energy scheduling model by considering equations (1)-(10) in our proposed hybrid $G^{2}$ DSM model.

\section{OPTIMIZATION METHODS}

Traditional optimization methods like integer linear programming (ILP), mixed integer programming (MILP) and mixed integer nonlinear programming (MINLP) are unable to control large number of appliances. Furthermore, these methods are computationally inefficient and hence not suitable for real time optimization, which is deterministic in nature. Instead, the meta-heuristic optimization technique can provide a best solution while considering user defined constraints. We are applying genetic algorithm (GA), grey wolf optimization (GWO) method and a hybrid of both techniques to achieve real time optimal results.
GA is inspired from the genes of living organisms. Initially, binary coded chromosomes are randomly initialized. Total number of smart appliances are represented by the length of chromosomes' and smart appliances ON/OFF status is identified through chromosomes binary coded pattern. Once the initial population is generated, fitness function of GA is evaluated which is actually an objective function of this study. Mutation and crossover are performed to generate new population. Generated population fitness function is then compared with the previous one and hence, optimum results are achieved.

On the other hand, GWO algorithm is based on grey wolves hunting and leadership hierarchy mechanism. Alpha, beta, delta and omega are four kinds of wolves in leadership hierarchy. For performing optimization hunting, searching, encircling and attacking, prey steps are implemented. In this way, position of the search agents are updated in the form of position vector towards prey. Search agents update its position until it reaches to an optimal position in n-dimensional search space.

The purpose of proposing the hybrid technique is to achieve a balance between global search and local search. GA performs well in terms of exploration mode. Also, it has good convergence rate to reach to optimal solution. Initially, GA steps are followed for generating initial population of chromosomes. These chromosomes actually represent candidate solution to the problem. Furthermore, a bit of chromosomes represents the ON/OFF state of the smart appliances. Fitness function is based on objective function, taken from GWO. The best population is regenerated through velocity updating step of GWO. Firstly, it finds a local best solution and on the basis of this value, it achieves a global best solution. Through an optimal stopping rule, the cost minimization problem can be formulated and the best fit value is thus chosen. Based on crossover and mutation, a new stream of is generated. Hence new generation population is created which has completely different characteristics as compared to the initial generation.

\section{SIMULATION RESULTS}

In this section, we present simulation results and assess the performances of the proposed algorithms. By making RTP signal for DSM, PAR reduction, cost minimization and load balancing are key features to be analyzed. The cost, load and waiting time for each group is represented in terms of cents, hours and kWh. Fig. 2 shows the load on grid for a single home using all three approaches according to RTP. In RTP tariffs, electricity price changes during different times of a single day. Particularly prices are higher in the afternoon, hot summer days and cold winter days. Fig. 3 clearly demonstrates that during the high price rate hours, if demand is high, then unscheduled load creates high peaks as compared to the scheduled load. Due to this reason, electricity cost of unscheduled load is high. It also depicts that without affecting the overall load, the proposed fitness function has a greatest effectiveness on cost and PAR reduction.

Moreover, load profile during multiple time slots for a 
complete day is shown in Fig. 4. It demonstrates that the proposed hybrid model outperforms the GA and GWO models in terms of load shifting to off-peak hours; hence reduction can be many folds in terms of PAR and cost. Fig. 4 illustrates the cost in different time slots during the day, the consumption pattern by GWO and GA during the peak price is high as compared to the hybrid $G^{2}$ approach. This affects the overall cost per day for aforementioned approaches as shown in Fig. 5. It clearly shows that price using hybrid $G^{2}$ is low as compared to GA and GWO. Using the hybrid $G^{2}$, the proposed approach reduces $20 \%$ cost, which is the best among all three used approaches

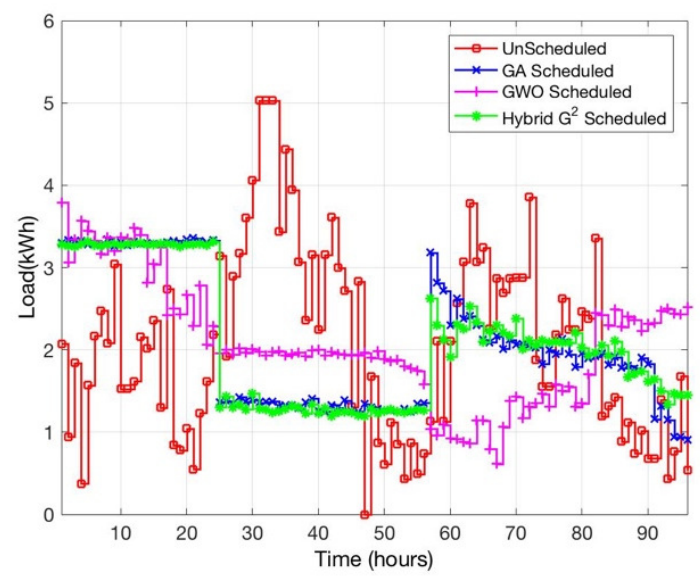

Fig. 2 Load profiles

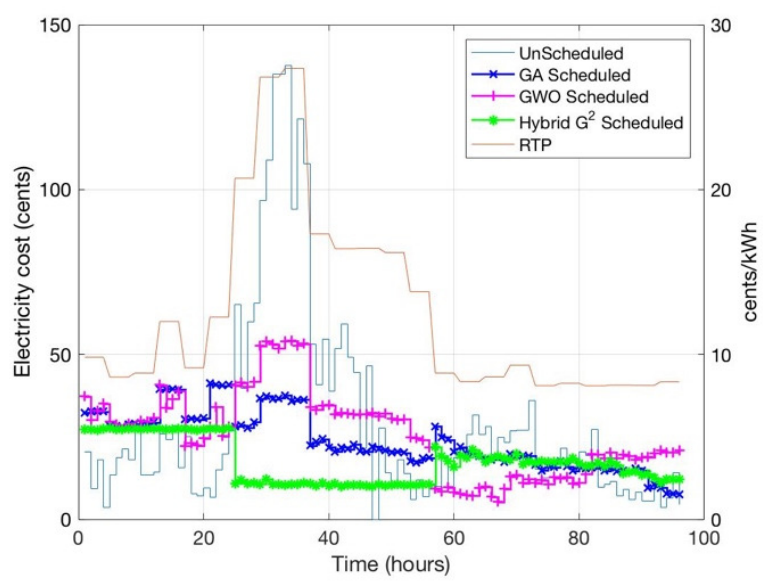

Fig. 3 Energy cost during the time slots

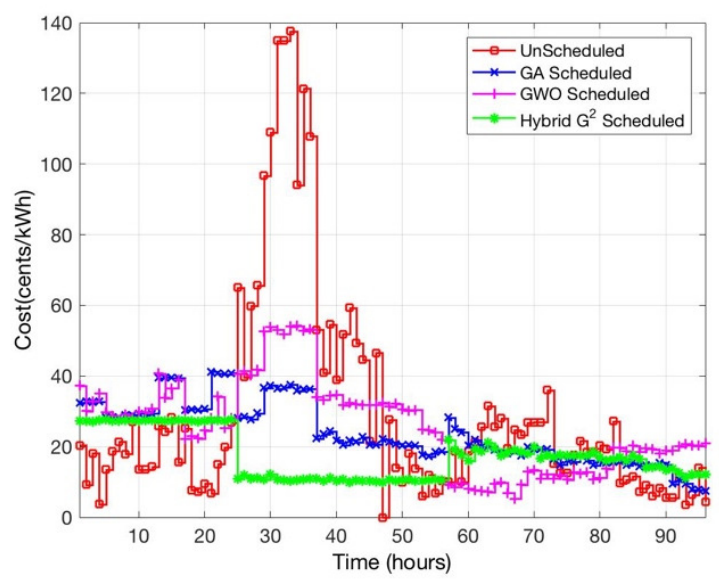

Fig. 4 Cost in different time slots over the day
PAR results are shown in Fig. 6, where the unscheduled load is very high and for the hybrid $G^{2}$ it is commendable. This shows adeptness of the proposed approach which is better than GA and GWO. In this case, about $50 \%$ PAR is reduced by hybrid $G^{2}$. While addressing the cost and PAR, waiting time of different appliances cannot be overlooked; this is highlighted in Fig. 7. Waiting time has a direct relationship and impact on user comfort and it is an important parameter for efficiency measurement in any proposed scheme. It shows that waiting time for base load appliances for GA and GWO is higher as compared to the hybrid $G^{2}$.

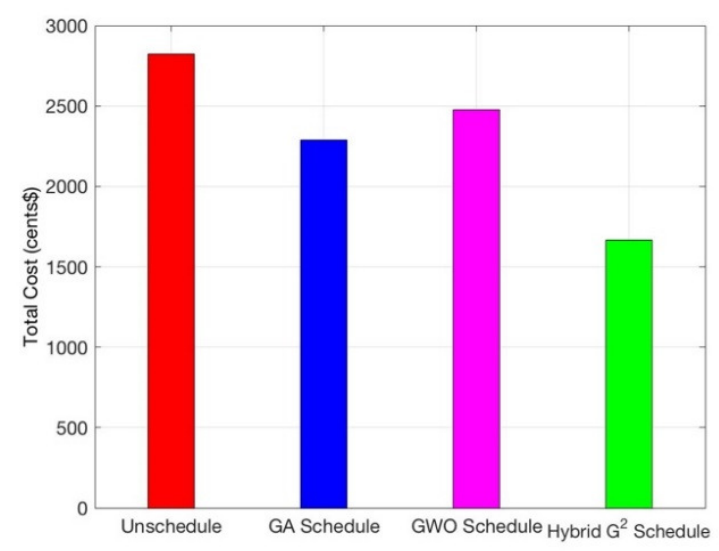

Fig. 5 Total cost under different approaches

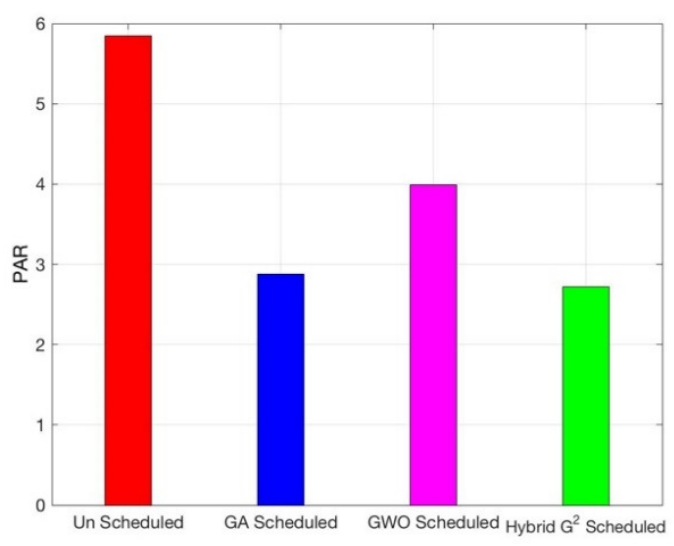

Fig. 6 PAR under different schemes

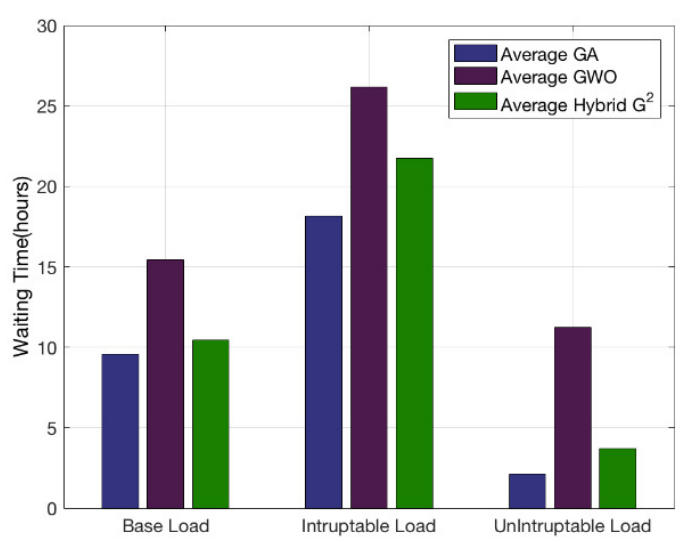

Fig. 7 Waiting time for the different approaches 
During the simulation, we perceived that GA is best for maximum number of populations. With the increase in number of population and generation step, difference among the lowest and highest point becomes negligible. On the other hand, GWO shows high performance for small population under hundred intervals. Fig. 5 and Fig. 6 show that GA outperforms GWO in terms of cost reduction, peak reduction and PAR. The hybrid $G^{2}$ shows positive influence on both approaches by lowering PAR, cost and peak load values.

\section{CONCLUSIONS}

In this paper, we have presented an effective approach for load management by shifting or balancing home appliances in an optimum way. The main idea is to facilitate consumers to reduce electricity cost. From the simulated results, it is observed that considerable saving in energy costs can be realized by consumers. To facilitate consumers, artificial intelligence based optimization technique is adopted. The results show that through a carefully designed appliance scheduling model, users can offer a viable solution to optimal power management among residential energy users. The proposed approach is based on a hybrid GA and GWO.

It clearly demonstrates that the hybrid approach outperforms the GA and GWO. The load is balanced in such a way that not only load peaks are avoided but also user comfort is less compromised. It is worth mentioning that there exists the tradeoff between cost and PAR. Since cost is minimized at certain time extents the load to offpeak hours using the proposed model, resulting in maximizing PAR. Results show the effectiveness of the proposed hybrid model in terms of cost minimization. Future work will consider integration and testing RES along with real-time pricing signal.

\section{ACKNOWLEDGEMENTS}

The authors would like to acknowledge the funding support from Lancaster University, UK and COMSATS University, Pakistan.

\section{REFRENCES}

[1] Wen, Zheng, Daniel O'Neill, and Hamid Maei. "Optimal demand response using device-based reinforcement learning." IEEE Transactions on Smart Grid 6.5 (2015): 2312-2324.

[2] Mohsenian-Rad, Amir-Hamed, et al. "Autonomous demand-side management based on game-theoretic energy consumption scheduling for the future smart grid." IEEE transactions on Smart Grid 1.3 (2010): 320-331.

[3] Shirazi, Elham, and Shahram Jadid. "Optimal residential appliance scheduling under dynamic pricing scheme via HEMDAS." Energy and Buildings 93 (2015): 40-49.

[4] Rahim, Sahar, et al. "Exploiting heuristic algorithms to efficiently utilize energy management controllers with renewable energy sources." Energy and Buildings 129 (2016): 452-470.
[5] Mesarić, Petra, and Slavko Krajcar. "Home demand side management integrated with electric vehicles and renewable energy sources." Energy and Buildings 108 (2015): 1-9.

[6] Deilami, Sara, et al. "Real-time coordination of plug-in electric vehicle charging in smart grids to minimize power losses and improve voltage profile." IEEE Transactions on Smart Grid 2.3 (2011): 456-467.

[7] Azar, Armin Ghasem, and Rune Hylsberg Jacobsen. "Appliance scheduling optimization for demand response." International Journal on Advances in Intelligent Systems $9.1 \& 2$ (2016): 50-64.

[8] Ramanathan, Badri, and Vijay Vittal. "A framework for evaluation of advanced direct load control with minimum disruption." IEEE Transactions on Power Systems 23.4 (2008): 1681-1688.

[9] Ahmad, Adnan, et al. "An optimized home energy management system with integrated renewable energy and storage resources." Energies 10.4 (2017): 549.

[10] Rasheed, Muhammad Babar, et al. "Priority and delay constrained demand side management in real-time price environment with renewable energy source." International Journal of Energy Research 40.14 (2016): 2002-2021.

[11] Ma, Kai, et al. "Residential power scheduling for demand response in smart grid." International Journal of Electrical Power \& Energy Systems 78 (2016): 320-325.

[12] Marzband, Mousa, et al. "Real time experimental implementation of optimum energy management system in standalone microgrid by using multi-layer ant colony optimization." International Journal of Electrical Power \& Energy Systems 75 (2016): 265-274.

[13] Lee, Jae Yong, and Seong Gon Choi. "Linear programming based hourly peak load shaving method at home area." Advanced Communication Technology (ICACT), 2014 16th International Conference on. IEEE, 2014.

[14] Nadeem, Zunaira, et al. "Scheduling appliances with GA, TLBO, FA, OSR and their hybrids using chance constrained optimization for smart homes." Energies 11.4 (2018): 888. 